\title{
Analysis and Research on Low-Cost and Non-Intrusive Power Metering Chip
}

\author{
Li-Ping Gao1,2, Xiu-Li Yu1,2, Liang Huang1,2, Tao-Rong Gong1,2 \\ ${ }^{1}$ State Grid Key Laboratory of Power Industrial Chip Design and Analysis Technology, Beijing Smart-Chip Microelectronics \\ Technology Co., Ltd. Beijing, China \\ ${ }^{2}$ Beijing Engineering Research Center of High-Reliability IC with Power Industrial Grade, Beijing Smart-Chip Microelectronics \\ Technology Co., Ltd. Beijing, China \\ Email: glp_mu@126.com,gaoliping@sgitg.sgcc.com.cn
}

How to cite this paper: Gao, L.-P., Yu X.-L., Huang, L. and Gong, T.-R. (2017) Analysis and Research on Low-Cost and Non-Intrusive Power Metering Chip. Energy and Power Engineering, 9, 573-580. https://doi.org/10.4236/epe.2017.94B063

Received: March 9, 2017

Accepted: March 30, 2017

Published: April 6, 2017

\begin{abstract}
Metering technology is one of the core technologies of the smart power grid. The overall metering solution and related products have a wide market space in the whole process of power production, which bring new opportunities for power distribution development from automation to intelligentialize, and provide technical supports for the power metering system platform. Because of the importance of metering products and their market demand, this paper focuses on the design of a simple power metering chip with low-cost, lowprecision and non-invasive, so as to lay the foundation for the development and practical technology accumulation of power metering products. The design achieves low cost by reducing the acquisition accuracy, simplifying the collection and sampling methods. This paper studies the chip accuracy, sampling methods, collection methods, and the inference of the chip characteristics requirements.
\end{abstract}

\section{Keywords}

Power Metering Chip, Low-Cost, Low-Precision, Sampling Methods

\section{Introduction}

With the rapid development of power system, the capacity of power grid increases, whose structure also becomes more and more complex. Thus, the automation of real-time monitoring and scheduling in power system is particularly important, while data acquisition is an important part of automation, especially how to collect the analog power signals accurately and quickly. Metering technology is one of the core technologies of the smart power grid. The overall metering solution and related products have a wide market space in the whole 
process of power production, which bring new opportunities for power distribution development from automation to intelligentialize and provide technical supports for the power metering system platform.

Metering products run through the whole process of power production. In the power system, generation, transmission, transformation, distribution, utilization, and adjustment are all involved in the applications of metering products, which plays an important role among power grid, source and load. The basic metering methods and collection technology are the fundamental guarantee for the relevant power metering enterprises to build metering product tree. The research of basic metering technology includes the calculation of basic parameters (such as E, P, U, I, etc.), and core algorithm (such as sampling and filtering). Only the core metering technology is handled, the further accuracy and consistency of metering product can be improved. At the same time, according to the energy characteristics of electric vehicle industry and high-speed rail industry, basic metering technology includes a range of research from the algorithms such as sampling, modeling and core controlling to providing a complete product solution, which can make a contribution to solving the DC energy measurement, impact load measurement, and the further development of measurement industry. Then, it makes a feasibility study on users' perception direction in the power grid, tracks and studies on the load identification technology, to lead the research in source-load interaction, sub-item measurement, etc.

Based on the importance of metering products and their market demand, this paper focuses on the design of a simple power metering chip with low-cost, low-precision and non-invasive, so as to lay the foundation for the development and practical technology accumulation of power metering products. Because of the limitation of cost and accuracy, simple outlines are collected among electrical information generally. Then the design achieves low cost by reducing the acquisition accuracy, simplifying the collection and sampling methods. This paper studies the chip accuracy, sampling methods, collection methods, and the inference of the chip characteristics requirements.

\section{Precision Positioning}

Under normal use conditions, the degree of deviation between the instrument metering result and the standard results is called the instrument accuracy [1]. If the reference error is smaller, the accuracy of the instrument will be higher. Then, the reference error is related to the range of the instrument. As a result, the instrument range is always compressed to reduce the measurement error. In industrial measurement, the accuracy level is used to facilitate the instrument accuracy, which is one of the important indicators to measure the quality of the instrument. The accuracy level can be obtained by the percentage value of the maximum reference error absolute value [2].

1) There are different values for industrial instrument accuracy level: 0.005 , $0.02,0.05,0.1,0.2,0.35,0.4,0.5,1.0,1.5,2.5,4.0$, etc. The smaller value means the higher accuracy. 
2) There are 16 values for the accuracy level of industrial process measurement, control instrument and indicating instrument: 0.01, 0.02, (0.03), 0.05, 0.1, $0.2,(0.25),(0.3),(0.4), 0.5,1.0,1.5,(2.0), 2.5,4.0,5.0$. The values in brackets are not recommended.

The power metering chip studied in this paper is mainly used in power system and related power distribution fields, such as electric trend monitoring, dispatching automation decision-making, power energy efficiency monitoring, power demand side management and so on. So the chip with a low accuracy (1 2 measurement accuracy level) can achieve the above goals, which is also a key factor why this chip can be low-cost.

\section{Analysis for Sampling Algorithm [3]}

\subsection{Sampling Methods}

According to the different sampling signals, the sampling can be divided into DC (Direct Current) sampling and AC (Alternating Current) sampling. DC sampling transforms the AC voltage and current signals into $0-5 \mathrm{~V}$ DC voltage to collect. The main advantage of this method is simple and easy to filter, but the disadvantage of large investment, complex maintenance and not achieving real-time signal acquisition, makes it limited in power system applications. AC sampling converts the alternating current quantity into $\pm 5 \mathrm{~V}$ (or $0-5 \mathrm{~V}$ ) AC voltage to collect. This method is with good real-time acquisition, small phase distortion, less investment and easy to maintain. But it is complex, difficult to improve the accuracy, and needing high A/D conversion speed. However, with the development of computer technology, AC sampling will be gradually replacing DC sampling with its excellent performance/price ratio. So the power metering chip in this paper takes AC sampling method.

\subsection{Analysis for AC Sampling Algorithm}

AC sampling is used widely, and depending on different applications, there are many kinds of AC sampling algorithm. According to the model function, it can be divided into sinusoidal model algorithm and non-sinusoidal period model algorithm. The former includes the maximum algorithm, single-point algorithm, half-cycle integral method, two-point sampling, etc.; the latter includes the root mean square algorithm, Fourier algorithm, etc. Various algorithms are applied in different power system applications with their advantages and disadvantages.

To collect the three-phase symmetrical sinusoidal signal, the single-point algorithm will be an ideal algorithm. This algorithm is accurate and fast, without depending on the sampling time, which can also get the voltage, current, active, reactive and other signals. But it requires high sampling signal, and its hardware is complex. For other algorithms, such as the maximum sampling, half integral sampling and two-point sampling, they are simple and fast, which can be completed in the half cycle. However, they have strict requirements for the input signals, only suitable for application with a sinusoidal signal or pre-filter device. The above sampling algorithms are fast and with good real-time performance, 
especially for relay protection system and real-time monitoring system [4].

The root mean square algorithm (RMS algorithm) can take into account the influence of high-order harmonics. If increasing the sampling points in each period, the sampling accuracy can be improved, but it leads to reducing sampling speed and increasing the computational complexity. Therefore, it needs to weigh and make a choice between the accuracy and speed.

The full-wave Fourier algorithm has a strong filtering ability, can collect signals in a variety of periodic volume, and calculate fundamental and high-order harmonic. On the other side, it has a low response speed, suitable for data acquisition when power calculation or other occasions with poor real-time but high precision requirements, which cannot satisfy the fast acquisition requirements. The recursive Fourier algorithm improves the response speed, but it has a delay effect, which will get a great error especially in the event of a sudden change in power.

To sum up, to meet the general measurement requirements and consider the measurement parameters may be further increased or expanded, the root mean square algorithm is the best choice, because it can handle any waveform acquisition and any parameter calculation, also calculate the waveform frequency without hardware capture circuit. The root mean square principle is analyzed as follows [5]:

Let

$$
\begin{gathered}
u=U_{m} \sin \omega t \\
U=\sqrt{\frac{1}{T} \int_{0}^{T} u^{2} d t}
\end{gathered}
$$

Discretize it,

$$
U=\sqrt{\frac{1}{N} \sum_{k-1}^{N} u_{k}^{2}}
$$

where $N$ is the number of equal intervals sampling per cycle and $u_{k}$ is the kth sample.

From the sampling theorem, this algorithm can take into account N/2-order harmonics.

Similarly,

$$
I=\sqrt{\frac{1}{N} \sum_{k-1}^{N} i_{k}^{2}}
$$

Discretize

$$
\begin{gathered}
P=\frac{1}{T} \int_{0}^{T} u \cdot i d t \\
P=\frac{1}{N} \sum_{k=1}^{N} u_{k} i_{k} \\
Q=\frac{1}{N} \sum_{k=1}^{N} u_{k} i_{\left(k-\frac{T}{\varphi}\right)}
\end{gathered}
$$




$$
S=U I
$$

\subsection{Analysis of Sampling Frequency and Sampling Points}

The highest analytical frequency: Fm refers to the highest frequency that needs to be analyzed, and also the highest frequency after the anti-aliasing filtering. According to the sampling theorem, generally, the relationship between Fm and the sampling frequency $F_{s}$ is [6]:

$$
F_{s}=2.56 F_{m}
$$

The choice of the highest analytical frequency depends on the speed of the instruments and the nature of the expected error.

Similarly, the number of sampling point $N$ has the following relationship with the number of spectrum line $M$ :

$$
N=2.56 M
$$

where, the number of spectrum line $M$ has the following relationship with the frequency resolution $\Delta F$ and the highest analytical frequency $F_{m}$ :

$$
\Delta F=F_{m} / M
$$

Which is

$$
M=F_{m} / \Delta F
$$

So

$$
\mathrm{N}=2.56 F_{m} / \Delta F
$$

Obviously, the number of sampling point is related to the frequency resolution. For example, the machine speed is $3000 \mathrm{r} / \mathrm{min}=50 \mathrm{~Hz}$, if the fault frequency is estimated below 8 times, and the frequency resolution on the spectrum is $\Delta F=1$, then the sampling frequency and sampling points are set to:

The highest analytical frequency

$$
F_{m}=8.50 \mathrm{~Hz}=400 \mathrm{~Hz}
$$

The sampling frequency

$$
F_{s}=2.56 \cdot F_{m}=2.56 \cdot 400 \mathrm{~Hz}=1024 \mathrm{~Hz}
$$

The number of the sampling point

$$
N=2.56 \cdot\left(F_{\mathrm{m}} / \Delta F\right)=2.56 \cdot(400 \mathrm{~Hz} / 1 \mathrm{~Hz})=1024
$$

The number of the spectrum line

$$
M=N / 2.56=1024 / 2.56=400
$$

From the above analysis, in this paper, the sinusoidal wave frequency is $50 \mathrm{~Hz}$, harmonic analysis is 21 times, and the highest analytical frequency is

$$
F_{m}=21 \cdot 50 \mathrm{~Hz}=1050 \mathrm{~Hz}
$$

The sampling frequency is

$$
F_{s}=2.56 \cdot F_{m}=2688 \mathrm{~Hz}
$$

In order to facilitate configuration and calculate easily, this paper assumes that it samples about 60 points per cycle, and its sampling frequency is $3 \mathrm{KHz}$. The 
filter algorithm samples 8 times each time using the same channel, and takes the intermediate 4 values to average. The sum of the squared data for one cycle is divided by the number of periodic sampling point $N$.

Where, the periodic sampling number is $N=3 \mathrm{KHz} /$ waveform frequency, and the frequency is calculated by sampling zero-crossing point.

In general, the metering chip sampling port input voltage is $0-5 \mathrm{~V}$. In general, the metering chip sampling port input voltage is $0-5 \mathrm{~V}$. When the test value does not exceed the $2 / 3$ range of instrument, the measuring precision is more accurate, that means sampling the waveform the peak value is $3.5 \mathrm{~V}$. The accuracy of 12 bit $\mathrm{AD}$ sampling chip can only reach the accuracy of 8 bit, so the above reference accuracy is $1 / 256=0.39$, about 0.4 , considering the influence of sampling data processing, the accuracy of the metering chip can reach the level 2.

\section{The Realization of Metering Chip}

The realization of the metering chip data processing functions, mainly rely on software program. According to the foregoing analysis, the sampling steps and related algorithms of current, voltage and frequency are followed.

1) Initialization: system clock (including $\mathrm{CPU}, \mathrm{AD}$, serial clock), $\mathrm{I} / \mathrm{O}$ port (input and output, sampling, serial port), interrupt (clear interrupt flag, mask global interrupt), AD (sampling mode, sampling channel), and Serial port (communication protocol, baud rate).

2) Data sampling and computation

a) Input 2 channels of voltage and current;

b) Filter the sampling value: adopt the average median filtering method (also known as anti-pulse interference average filtering method) and use 8 to 4 (that is, sort the 8 collected points, then remove the two maximum and two minimum values. In total $2 \times 8=16$ points needed);

c) Save and calculate the instantaneous sampling value of two channel signals: according to the collected data to determine the current, voltage zero-crossing points;

d) Compute the related data on zero-crossing point: the valid value, frequency and phase difference of two channel signals;

e) Set the sampling end flag;

f) If it crosses zero, set the zero flag; if not, clear the zero flag;

g) If interrupt, return.

3) Save data: save the processed sampling data

4) Data communication (subject to specific requirements)

5) Finish

Corresponding to the above steps, the software initialization program and sampling interrupt program flow chart is as the follows (Figure 1 and Figure 2).

\section{Ending}

The research of the basic measurement technology covers many things. On the 


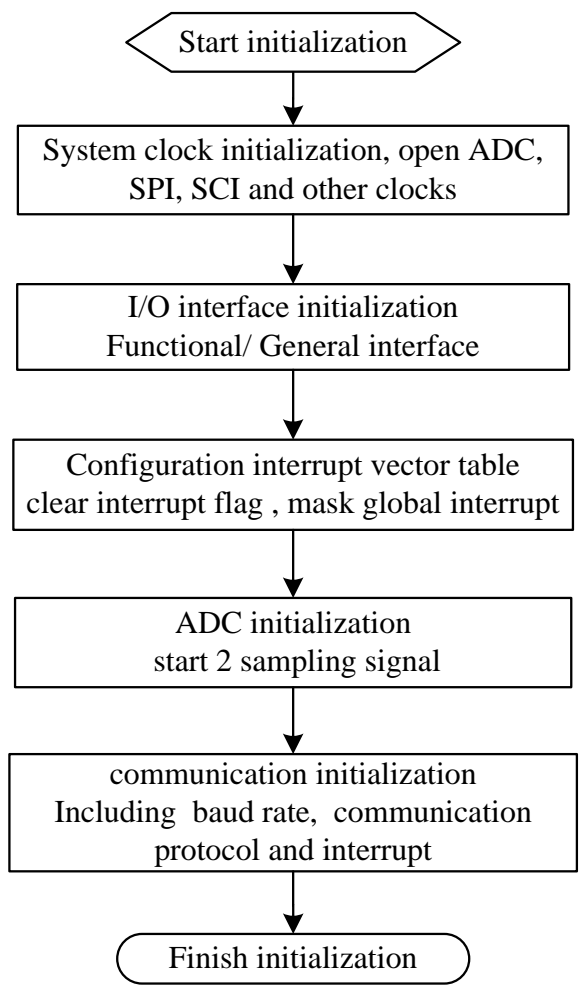

Figure 1. Initialization program.

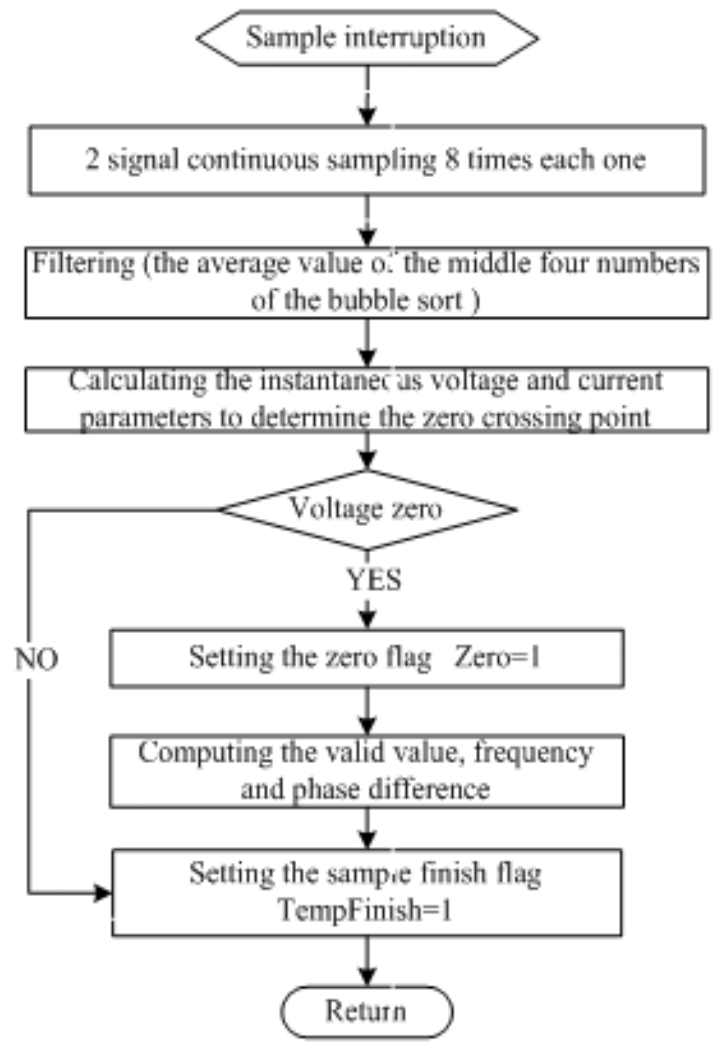

Figure 2. Sample interrupt program. 
one hand, the basic measurement technologies and methods need to be deeply understood through the practical application; the basic measurement technologies and related expertise should be further studied to complete the technology accumulation, inheritance and continuity. On the other hand, the basic measurement technologies mush be strengthen on the researched and transformation. Putting the research achievement into the metering chip is one of the tasks. How to reduce the cost, improve the accuracy level, and simplify the design of the power metering chip, is the entry point, also the key point to improve the product competitiveness.

\section{Acknowledgements}

Our chief engineer Zeng-Qiao Guo has made a careful examination of this paper and gave a lot of guidance. The scholar Li-Feng Gao has done a lot of work for the English translation of this paper. Thanks for your two jobs. The work is supported by Science and Technology project of State Grid Corporation of China (Grant No. SGHAZZ00FCJS1500238).

\section{References}

[1] Fang, Y.Z. (2007) Error Analysis of Calibrating Device for Electric Quantity Transmitter. North China Electric Power, 12, 13-16.

[2] Ge, F., Wang, Y.F., Hua, C. and Zhao, J.H. (2005) Study on the Detection of the Degree of Accuracy for the Weighing Sorting Machine. Machinery Design and Manufacture, 9, 104-105.

[3] (1994) Comparison of Normal Alternating Sampling Methods in Electric Power System. North China Electric Power, 4.

[4] Tie, S.G. and Ping, S. (2006) Analysis of the Problems in the Application of AC Sampling and Measuring Device. China Power System Protection and Control Symposium 2006.

[5] Lu, Y.L. (2005) Application of AC Sampling Measurement Device in Power System. Power Equipment, 6, 17-18.

[6] Zhou, Z.H. (2009) Research and Design of Power System Transient Power Collection Technology, Thesis, Guangxi University 2009, Guangxi. 
Submit or recommend next manuscript to SCIRP and we will provide best service for you:

Accepting pre-submission inquiries through Email, Facebook, LinkedIn, Twitter, etc. A wide selection of journals (inclusive of 9 subjects, more than 200 journals)

Providing 24-hour high-quality service

User-friendly online submission system

Fair and swift peer-review system

Efficient typesetting and proofreading procedure

Display of the result of downloads and visits, as well as the number of cited articles Maximum dissemination of your research work

Submit your manuscript at: http://papersubmission.scirp.org/

Or contact epe@scirp.org 